\title{
The Long-Term Effect of Early Anti-Tumor Necrosis Factor on Restoration of Growth in Pediatric Crohn's Disease
}

\author{
Jung Ok Shim ${ }^{1}$ and Yoon Tae Jeen ${ }^{2}$ \\ ${ }^{\prime}$ Division of Pediatric Gastroenterology, Hepatology, and Nutrition, Department of Pediatrics, Korea University Guro Hospital, Korea University \\ College of Medicine, and ${ }^{2}$ Division of Gastroenterology and Hepatology, Department of Internal Medicine, Korea University Anam Hospital, \\ Korea University College of Medicine, Seoul, Korea
}

See “Early Infliximab Yields Superior Long-Term Effects on Linear Growth in Pediatric Crohn's Disease Patients” by Jaeyoung Choi, et al. on page 255, Vol. 12. No. 3, 2018

Impairment of growth is one of the major complications in pediatric Crohn's disease (CD). This is a distinctive feature compared to adult $\mathrm{CD}$, and it may affect later health in adulthood. Impairment of growth at diagnosis has been reported in $4 \%$ to $38 \%$ of children with $\mathrm{CD}$, and growth velocity may be reduced in up to $88 \%$ of these children. ${ }^{1}$ In a recent study, growth failure was present in $8 \%$ of patients at diagnosis and still in 5\% of patients at final height. Children with $\mathrm{CD}$ have multiple risk factors for impaired growth, including, impaired bone modeling, delayed puberty, malnutrition, decreased muscle mass, and decreased physical activity. ${ }^{2}$ Insufficient food intake related to loss of appetite, intestinal malabsorption due to small bowel involvement of the disease, increased nutritional losses including protein losing enteropathy, iron, and zinc losses, and increased energy requirements may contribute to growth failure. A recent Korean study revealed that low vitamin D levels affect growth outcome in children with $\mathrm{CD} .^{3}$ Children with long-term use of steroids are at risk for growth failure. Moreover, children with CD with uncontrolled inflammation showed a lower growth velocity. ${ }^{1,4}$ Tumor necrosis factor $\alpha$ (TNF- $\alpha$ ) has direct negative effects on bone metabolism and plays an important role in CD pathogenesis. ${ }^{2}$

Numerous studies have reported the effect of anti-TNF- $\alpha$ agents on growth restoration. The REACH study revealed improvement of height $\mathrm{z}$-score (0.5) at week 54 with infliximab therapy, while initial bone age was delayed at least 1-year ( $\mathrm{z}$ score of -1.5). ${ }^{5}$ The REACH open-label extension study showed that the median height $\mathrm{z}$-score had improved by 1.56 at the end of 3 years of infliximab therapy. ${ }^{6}$ In a study of children with CD with a median 43-month follow-up, increases in lean massheight z-score and fat mass-height z-score were associated with infliximab therapy. Height-z score was not improved during this study period. ${ }^{7}$ Another study showed that 1 year of anti-TNF- $\alpha$ therapy was associated with improvements in trabecular bone mineral density and cortical structure. ${ }^{2}$

Several studies have considered early infliximab administration, before the use of corticosteroids. The recent guidelines of the European Crohn's and Colitis Organization and European Society for Pediatric Gastroenterology, Hepatology, and Nutrition state that infliximab may be considered as a primary induction regimen in selected children with high risks, which include marked growth retardation of $>-2.5$ height z-score. ${ }^{8}$ There is lack of data to evaluate the efficacy of early use of infliximab in relation to growth restoration. Recently Church et al. ${ }^{9}$ reported that children with height was normalized (mean z-score 0) by 3 years in children with Tanner stage 1 to 2 for whom infliximab was initiated early, within 18 months of diagnosis, whereas those for whom infliximab was initiated after a longer duration remained shorter than healthy children.

In this issue of Gut and Liver, the article "Early infliximab yields superior long-term effects on linear growth in pediatric Crohn's disease patients" by Choi et al. ${ }^{10}$ investigated the efficacy of early combined immunosuppression on restoration of growth compared to conventional "step-up" therapy for a long-term period of 3 years. This retrospective study included 33 children with moderate to severe $\mathrm{CD}$ who had sustained

Correspondence to: Yoon Tae Jeen

Division of Gastroenterology and Hepatology, Department of Internal Medicine, Korea University Anam Hospital, Korea University College of Medicine, 73 Inchon-ro, Seongbuk-gu, Seoul 02841, Korea

Tel: +82-2-920-6555, Fax: +82-2-953-1943, E-mail: ytjeen@korea.ac.kr pISSN 1976-2283 eISSN 2005-1212 https://doi.org/10.5009/gnl18112

(c) This is an Open Access article distributed under the terms of the Creative Commons Attribution Non-Commercial License (http://creativecommons.org/licenses/by-nc/4.0) which permits unrestricted non-commercial use, distribution, and reproduction in any medium, provided the original work is properly cited. 
clinical remission with combined immunosuppression therapy including infliximab and azathioprine. Infliximab was initiated at a median 0.7 months after diagnosis in the early combined immunosuppression group induced, whereas it was initiated 11.4 months after diagnosis in the step-up group. The median age at diagnosis was significantly lower in the step-up group compared to the early immunosuppression group (12.1 years vs 15.0 years, $p=0.009$ ). Though the distribution of height $z$-scores was not different between the two groups, further analysis with generalized estimating equations showed the interaction effect between time and treatment strategy for height and weight $\mathrm{z}$ score ( $p=0.026$ and $p=0.031$, respectively) in the early combined immunosuppression group compared to in the step-up group after adjusting for sex, age at diagnosis, and Tanner stage at diagnosis. This difference was representative in a subgroup with Tanner stage 1 to 2, whereas no difference was found in children with Tanner stage 4 to 5 .

This result is consistent with the recent study by Church et al. ${ }^{9}$ which showed that earlier use of infliximab was more effective for catch-up of a height z-score. Moreover, this is the first report on the long-term effect of early (within 1 month from diagnosis) combined immunosuppression therapy on linear growth. Previous studies also reported that a restoration of linear growth in children with CD was more likely to be found in pre- or earlypubertal stage children, who had growth potential.,4,9

This retrospective study includes the possibility of selection bias. All enrolled children in the early combined immunosuppression therapy group were in clinical remission during the study periods, and thus had expected catch-up growth. Children in the step-up group experienced relapse or no response to corticosteroid therapy before initiating infliximab (median, 11 months). This suggests that they might have periods of uncontrolled immune status, which may have an adverse effect on the restoration of growth. On the other hand, children in the step-up group had a younger median age, thus they may have a higher growth potential. It may neutralize the difference between the two treatment groups. Furthermore, loss of response and adverse effects should be considered when we are considering early initiation of infliximab.

Despite some limitations, this first investigation is very important, because it shows the possible positive effects of early introduction and long-term maintenance of combined immunotherapy on catch-up growth in children with $\mathrm{CD}$. This study inspires further large-scale randomized controlled studies of pediatric $\mathrm{CD}$.

\section{CONFLICTS OF INTEREST}

No potential conflict of interest relevant to this article was reported.

\section{REFERENCES}

1. Ley D, Duhamel A, Behal H, et al. Growth pattern in paediatric Crohn disease is related to inflammatory status. J Pediatr Gastroenterol Nutr 2016;63:637-643.

2. Griffin LM, Thayu M, Baldassano RN, et al. Improvements in bone density and structure during anti-TNF-alpha therapy in pediatric Crohn's disease. J Clin Endocrinol Metab 2015;100:2630-2639.

3. Lee EJ, Moon JS, Ko JS, et al. Effect of the baseline vitamin D level on growth outcome in pediatric Crohn disease. Pediatr Gastroenterol Hepatol Nutr 2017;20:41-46.

4. Cameron FL, Altowati MA, Rogers P, et al. Disease status and pubertal stage predict improved growth in antitumor necrosis factor therapy for pediatric inflammatory bowel disease. J Pediatr Gastroenterol Nutr 2017;64:47-55.

5. Hyams J, Crandall W, Kugathasan S, et al. Induction and maintenance infliximab therapy for the treatment of moderate-to-severe Crohn's disease in children. Gastroenterology 2007;132:863-873.

6. Hyams J, Walters TD, Crandall W, et al. Safety and efficacy of maintenance infliximab therapy for moderate-to-severe Crohn's disease in children: REACH open-label extension. Curr Med Res Opin 2011;27:651-662.

7. Thayu M, Denson LA, Shults J, et al. Determinants of changes in linear growth and body composition in incident pediatric Crohn's disease. Gastroenterology 2010;139:430-438.

8. Ruemmele FM, Veres G, Kolho KL, et al. Consensus guidelines of ECCO/ESPGHAN on the medical management of pediatric Crohn's disease. J Crohns Colitis 2014;8:1179-1207.

9. Church PC, Guan J, Walters TD, et al. Infliximab maintains durable response and facilitates catch-up growth in luminal pediatric Crohn's disease. Inflamm Bowel Dis 2014;20:1177-1186.

10. Choi J, Kang B, Kim MJ, Sohn I, Lee HJ, Choe YH. Early infliximab yields superior long-term effects on linear growth in pediatric Crohn's disease patients. Gut Liver 2018;12:255-262. 